\title{
Quantitative interactions between Candida albicans and the mutans streptococci in patients with Down Syndrome
}

\author{
Alfredo G. Linossier ${ }^{1}$, Benjamín Martinez ${ }^{2}$, Carlos Y. Valenzuela ${ }^{3}$ \\ ${ }^{1}$ Department of Dentistry and Oral Maxilo facial Surgery, School of Medicine, Pontificia Universidad Católica de Chile \\ ${ }^{2}$ Oral Pathology, School of Dentistry, Faculty of Sciences, Universidad Mayor. Santiago, Chile \\ ${ }^{3}$ Faculty of Medicine, Institute of Biomedical Sciences. Human Genetics Program. Universidad de Chile
}

Correspondence:

Gran Avenida 6477, Depto. 208

Santiago, Chile

alinossi@gmail.com

Received: $18 / 04 / 2020$

Accepted: $28 / 10 / 2020$

Linossier AG, Martinez B, Valenzuela CY. Quantitative interactions between Candida albicans and the mutans streptococci in patients with Down Syndrome. Med Oral Patol Oral Cir Bucal. 2021 Jan 1;26 (1):e1-7.

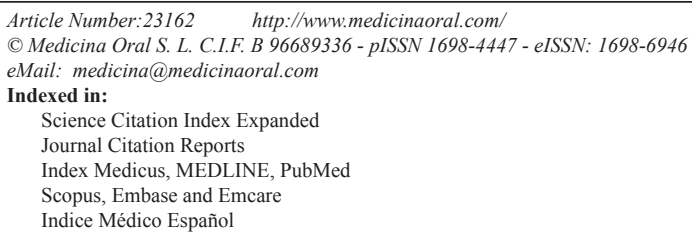

\begin{abstract}
Background: Oral microorganisms produce damage through the transfer to bloodstream, colonizing other tissues or direct damage in the oral cavity. Aim to study the quantitative interactions between C. albicans and the mutans streptococci and ms serotypes in the saliva of the oral cavity of patients with Down syndrome (DS).

Material and Methods: Included 120 patients of both genders, 60 patients with Down syndrome (DS) and 60 patients as a control group (CG). Samples of saliva were taken, and bacteria and fungi were grown on TYCSB and Saboureaud agar. Microbiological, serological and quantitative analyses were performed to determine the kind of isolated of microorganisms corresponding to the ms c, e, f and $\mathrm{k}$ for species $S$. mutans and d and $\mathrm{g}$ for S. sobrinus and $C$. albicans. Electronic scanning microscopy was employed to visualize and confirm the colonies under study. Statistics analysis included t-test proofs for matched data test, Scheffé and ANOVA.

Results: Forming units (CFU) per $\mathrm{mL}$ of saliva of C. albicans a significant difference was observed among DS $>\mathrm{CG}$ groups. A correlation of the $C$. albicans quantity and the ms count was found by age intervals however, tendencies were different in SD and CG. Also, the CFU of C. albicans was different among the serotypes of $\mathrm{ms}(\mathrm{c}, \mathrm{e}, \mathrm{f}, \mathrm{k}<\mathrm{d}$, $\mathrm{g}, \mathrm{h},<$ notyped).

Conclusions: These results show a significant non-random association between these two commensal microorganisms in different patient groups.
\end{abstract}

Key words: Down Syndrome, Candida albicans, microorganism interactions, mutans streptococci and oral cavity. 


\section{Introduction}

The oral cavity is colonized by different microorganisms in their different structures such as mucosa, teeth and saliva (1). These microorganisms constitute the biofilm, in which they grow and develop depending on the conditions of the environment if it is favorable to him and of the conditions given by the same microorganisms and the guest (2). Among these are the metabolic factors of the host and the microorganisms, chemical signals (quorum sensing) typical of them and the toxins they produce. The oral cavity research is oriented towards a model of interaction between the microorganisms that make up the community (3). During the first stages of the formation of the biofilm, bacterial cells are organized in plantonic phase rushing the surfaces of the oral cavity or indirectly bind to other bacterial cells that have already been colonized (4). This last point is confirmed by a population study of ancestral dental calculus, which shows different morphotypes of microorganisms (5). In research of congregation between yeasts and oral bacteria, there has been evidence of the presence in vitro of streptococci and C. albicans (6). In other studies that have used a specific fluorescein-labeled oligonucleotides probe (FITC), they allowed investigators to observe a congregation through microscopy, visualizing as a maize corn cob form where the center was occupied by C. albicans surrounded by species of streptococcus (7). In a study model in vivo, the possible association was shown between S.mutans and C.albicans in caries production in children at an early age (8). The C.albicans is part of the biofilm, giving to it the modulation of the different species in terms of the quality and quantity of them that make up micro environment of the oral cavity (9). Thus we will study the interaction between the serotypes of mutans streptococci (ms) and fungus Candida albicans, as secondary colonizers of the biofilm. They have been described as alterations in the oral cavity, and from there they can produce disturbances at the systemic level by these microorganisms. So we have the ms that includes different species in the human and within it the $S$. mutans, where it is possible to recognize serotypes such as $c, e, f$ and $k$ for $S$. mutans and $\mathrm{d}, \mathrm{g}, \mathrm{h}$, for S.sobrinus (10). We know that $\mathrm{ms}$ has a great prevalence in the oral human cavity, with a range of detection for S. mutans between 74 and $90 \%$ while for S.sobrinus the prevalence is lower (11). C albicans and S.mutans are considered commensal microorganisms, coexisting as homeostatic mutualism. When the balance is altered, due to environmental changes occurring in the mouth or by guest alterations at the systemic level, they become pathogens (12). So, we have that the $S$. mutans is a microorganism associated with tooth decay (11). In relation to dental caries in patients with Down Syndrome the evidence is limited and controversial (13). At a systemic level the oral streptococci produces infectious endocar- ditis $20 \%$ (14). It affects $71 \%$ the endocarditis subacute presenting serotype $\mathrm{k}$, retrieved from the heart valves (15). This possesses proteins (CBPs) approximately 120 $\mathrm{KDa}$, named $\mathrm{cnm}$ and $\mathrm{cbm}$. These surface antigens relate to the adhesion of the S.mutans to collagen tissue (16). As to C. albicans, its virulence is determined by its adherence to the surfaces of internal tissues in 5\% , which occurs in several stages; the infection is due to the presence of a protein ALS3 (17). It should be kept in mind that patients with Down syndrome show multiple systemic deficiencies. In the oral cavity, both humoral and cellular immunologic responses are involved, the latter being the most impaired of the cell-mediated immune system, leading to increased autoimmune diseases $(18,19)$. Previous studies have shown a quantitative relationship of the commensalism between S. mutans and $C$. albicans and the serotypes distributed between patients with DS and CG $(20,21)$.

The objective is to quantify the interaction in the saliva of $C$. albicans and the mutans streptococci in patients with DS and CG.

\section{Material and Methods}

- Subjects

During 2000 and 2001.This study was carried out in 120 males and females children and adolescent, aged 5 to19 years, were studied. The belonged to the public schools of the southern of the Metropolitan Region of Santiago. Among them 60 presented Down Syndrome (DS) and 60 were control group (CG). These data were obtained by observing ethical protocols at the time of collection. Patients who received antibiotics for less than 21 days before examination were excluded.

- Saliva samples

Were collected according to the following protocol: Two hours after breakfast a dentist brushed the patient's teeth for thirty seconds. Salivary flow was stimulated by applying a $1 \%$ solution of citric acid on the dorsal side of the tongue. After one minute, each patient's samples were collected using a glass funnel and kept at $0{ }^{\circ} \mathrm{C}$ for microbiological analysis. The volume of saliva collected was at least $0.5 \mathrm{ml}(20)$.

- Microbiological samples

The saliva is homogenized in a Vortex mixer (Max MixII tipo 37600 Mixer) for $60 \mathrm{~s} ; 100 \mu \mathrm{l}$ of saliva were added to $900 \mu \mathrm{l}$ of a Na2 HPO4 0,2M buffer solution (Sigma, St Luis,MI, EEUU)). The resulting solution was again homogenized by sonication for $2 \mathrm{~min}$ at $37^{\circ} \mathrm{C}$ and a volume of $100 \mu \mathrm{l}$ were streaked in an agar plate with TYCSB (22). The plates were incubated using an anaerobic system (Gas Pack jars) with a mixture of $95 \%$ $\mathrm{N} 2,5 \% \mathrm{CO} 2$, for $48 \mathrm{~h}$ at $37^{\circ} \mathrm{C}$.

The colonies were counted according to the method described by Westergreen and Krasse. The adherent colonies of ms were observed by transillumination in a 
Spencer magnification lens $(10 \mathrm{x})$. The total number of Streptococci colonies present in the Petri plate where obtained using the dilution coefficient and were called colony-forming units per $\mathrm{mL}$ of saliva $(\mathrm{CFU} / \mathrm{mL})$. As for $C$. albicans, they were obtained in agar Sabouraud, which were incubated in aerobic conditions for $48 \mathrm{~h}$ to $37 \mathrm{o}(20)$. - Biochemical study

The biochemical identification of $S$. mutans, or S.sobrinus was done by inoculating 2 colonies in the Todd Hewitt broth [3.1 g Brain Heart Infusion, 20g Peptone,2g Glucose, $2 \mathrm{~g} \mathrm{Na} \mathrm{Cl}$ 0,4g Na2 HPO4, 2,5g $\mathrm{Na} 2 \mathrm{CO} 3]$ during 18 hours. Bacteria were collected by centrifugation at 5,000 rpm. for $5 \mathrm{~min}$.The pellet was resuspended in 0,2 M Na2 HPO4 $(7,4)$ buffer at N0 5 Mac Farland units (1.5x $109 \mathrm{CFU})$. This suspension was used to identify biotypes of smg through the following micro method: in a sterile plastic box was place a rectangle glass of $11.5 \times 8 \mathrm{~cm}$, in 48 pieces of $0.8 \mathrm{~cm}$ in diameter each one, and the $30 \mu \mathrm{l}$ suspension for carbohydrate was incubated in a cell culture chamber for 18 hours. The yeasts were identified as $C$. albicans based on formation of germinal tube that appears in human plasma when the cell incubate for 2 hours $37^{\circ} \mathrm{C}$ and carbohydrate assimilation test $(20,21)$.

- Scanning electron microscopy

To reveal the adhesion of ms to yeast cells of C. albicans, the method described by Holmes et al. was used. Cellular aggregations, previously centrifuged (6000 X5 min) were set Glutaraldehyde to $2.5 \%$ (vol/vol) in buffer of sodium cacodilato at $0.1 \mathrm{M}$ at $4^{\circ} \mathrm{C}(\mathrm{pH} 7.4)$ for $90 \mathrm{~min}$. Cells were harvested by centrifugation for $1 \mathrm{~min}$ at $12000 \mathrm{x} \mathrm{g}$ and washed 4 times in $0.1 \mathrm{M}$ sodium cacodylate. After this, cell were fixed with $1 \%$ osmium tetroxide at 20 o C (1 h). Samples were dehydrated in different serial concentration of ethanol (30, 50,70, 95 an 100\%) and dried with CO2 using a critical point apparatus (Polaron England) Samples were examined in a Zeiss DSM 940 (15 KV) (20). - Serological study

It was performed using the double immunodiffusion technique described by Ouchterlony. The antiserum was prepared in female rabbits immunized with $S$. mutans (Ingbritt, serotype c) and S. sobrinus (OMZ 176 serotype d). The antigen was extracted by heat for 30 min at $60^{\circ} \mathrm{C}(21-24)$. The strains were kindly provided by Professor Bratthall (RIP) of Sweden and Professor Loesche of the United States. For C.albicans was used as Reference strain ATCC 10231.

- Statistical analysis

To determine the differences between age groups in the count of $C$. albicans, ms and their serotypes, these groups where analyzed using one way ANOVA. In addition, the Scheffé test was used for comparison between groups and the non-paired T-Test to confront the control group with the Down syndrome Group. Previously the Shapiro-Wilks test was used to determine if there was a normal distribution of the sample. The analysis was performed with software Stata v 14.1 and it was considered that there were significant differences if $p$ value $<0.05$.

\section{Results}

The sample analysis of Colony count/ml between CG and DS between $C$. albicans and ms proved to be significant for C.albicans $(P<0,005)$ (Table 1).

When performing the linear regression analysis between patients and comparing the $\log$ of $C$. albicans versus $\log \mathrm{ms}$, and confronting these slopes of both groups CG vs DS, we observed a significant differences $(p<0,005)$ (Fig. 1). Patients with Down Syndrome presented higher numbers (colonies/ml of saliva) of C. albicans and $\mathrm{ms}$ (colonies/ml saliva) than the CG in their respective means of cultivation of the same saliva sample. Comparison of the average C. albicans and ms ( $\log /$ $\mathrm{ml}$ ) acording to age between control group and Down Syndrome finding significant different for ms GC and Down Syndrome groups age between 5 to 9 and 10 to 14 and 10 to 14 and 15 to 19 years $(P<0,005)$ and $(P<0,002)$ (Table 2), there were no significant differences in counting in for C. albicans in GC and SD.

Table 1: Comparison of the log count in saliva (colonies $/ \mathrm{ml}$ ) in Control group and Down Syndrome, between mutans streptococci and Candida albicans.

\begin{tabular}{|l|l|c|c|}
\hline Group & $\mathbf{n}$ & $\begin{array}{c}\mathbf{m s} \\
\mathbf{x} \pm \mathbf{d s}\end{array}$ & $\begin{array}{c}\text { Candida albicans } \\
\mathbf{x} \pm \mathbf{d s}\end{array}$ \\
\hline Control group & 60 & $4,16 \pm 1,21$ & $3,20 \pm 0,57$ \\
\hline Down Syndrome & 60 & $4,35 \pm 1,73$ & $4,23 \pm 1,05$ \\
\hline & & $\mathrm{T}^{*}=0,689 ;$ & $\mathrm{T}^{*}=0,689 ;$ \\
& & $P=0,4934$ & $P<0,005$ \\
\hline
\end{tabular}

*Non-paired t Test.

Table 2: Comparison of saliva (colonies/ml) de ms (log) in Syndrome Down and control groups.

\begin{tabular}{|l|l|c|c|}
\hline Age (year) & $\mathbf{n}$ & $\begin{array}{c}\text { Syndrome Down } \\
\mathbf{x} \pm \mathbf{d s}\end{array}$ & $\begin{array}{c}\text { Control group } \\
\mathbf{x} \pm \mathbf{d s}\end{array}$ \\
\hline 5 a 9 & 20 & $4.52 \pm 1,87^{*}$ & $3,52 \pm 0,55^{*}$ \\
\hline 10 a 14 & 20 & $3,33 \pm 1,64^{*} \neq$ & $5,06 \pm 1,04^{*} \neq$ \\
\hline 15 a 19 & 20 & $5,20 \pm 1,87 \neq$ & $3,92 \pm 1,35 \neq$ \\
\hline & & $P<0,002$ & $P=0,005$ \\
\hline
\end{tabular}

$* \neq$ Anova and Test Scheffé 
There were not significant age differences for $C$ albicans in CG and SD according ages in groups 5 to 9 and 10 to 14 and between 10 a 14 and 15 to 19 (Table 3).

Analyzed the average count for $C$. albicans found in the two groups of patients studied and the serotypes of the ms. (Table 4). When performing t-Test comparing both patient groups for serotypes, it was significant for serotypes $\mathrm{c}$, e and $\mathrm{f}(p<0,005)$ and serotypes $d$ and $g(p<$ $0,02)$. No-typed was only observed in DS.
It was observed through scanning electron microscopy (SEM), colonies in a sample of saliva of patients, using as culture medium TYCSB for the ms, standing out yuxtaposition of $C$. albicans in the culture medium (Fig. 2). When quantification was carried out in agar Saboureaud medium, an increase in the concentration was observed about between 104-105 Colonies/ml in saliva for $C$ albicans, comparable to TYCSB that there was an increase in ms in patients with Down Syndrome (Fig. 2).

Table 3: Comparison of Candida albicans saliva count (log) in Syndrome Down and Control Group.

\begin{tabular}{|l|c|c|c|}
\hline Age (year) & $\mathbf{n}$ & $\begin{array}{c}\text { Syíndrome Down } \\
\boldsymbol{x} \pm \mathbf{d s}\end{array}$ & $\begin{array}{c}\text { Control Group } \\
\mathbf{x} \pm \mathbf{d s}\end{array}$ \\
\hline $\mathbf{5}$ a 9 & 20 & $4,30 \pm 1,23$ & $3,11 \pm 0,18$ \\
\hline $\mathbf{1 0}$ a 14 & 20 & $4,21 \pm 1,02$ & $3,26 \pm 0,55$ \\
\hline $\mathbf{1 5}$ a 19 & 20 & $4,18 \pm 0,93$ & $3,22 \pm 0,82$ \\
\hline & & $P<0,094$ & $P=0.712$ \\
\hline
\end{tabular}

Table 4: Average count of Candida albicans between patients in the control group (CG) and patients with Down Syndrome. Presents the serotypes for ms c, e, f, S.sobrinus d, g, and no type (only present in Down Syndrome).

\begin{tabular}{|c|c|c|c|}
\hline & \multicolumn{3}{|c|}{ Serotype } \\
\hline Group & $\begin{array}{c}\boldsymbol{c}, \boldsymbol{e}, \boldsymbol{f}(\boldsymbol{n}=\mathbf{6 0}) \\
\boldsymbol{x} \pm \mathbf{d s}\end{array}$ & $\begin{array}{c}\boldsymbol{d , g}(\boldsymbol{n}=\mathbf{6 0}) \\
\boldsymbol{x} \pm \mathbf{d s}\end{array}$ & $\begin{array}{c}\text { no type }(\boldsymbol{n}=\mathbf{6 0}) \\
\pm\end{array}$ \\
\hline Control Group & $3,15 \pm 0,54$ & $3,67 \pm 0,78$ & - \\
\hline Down Syndrome & $4,1 \pm 1,08$ & $4,93 \pm 0,55$ & $4,57 \pm 0,94$ \\
\hline & $P<0.05$ & $P<0.02$ & \\
\hline
\end{tabular}

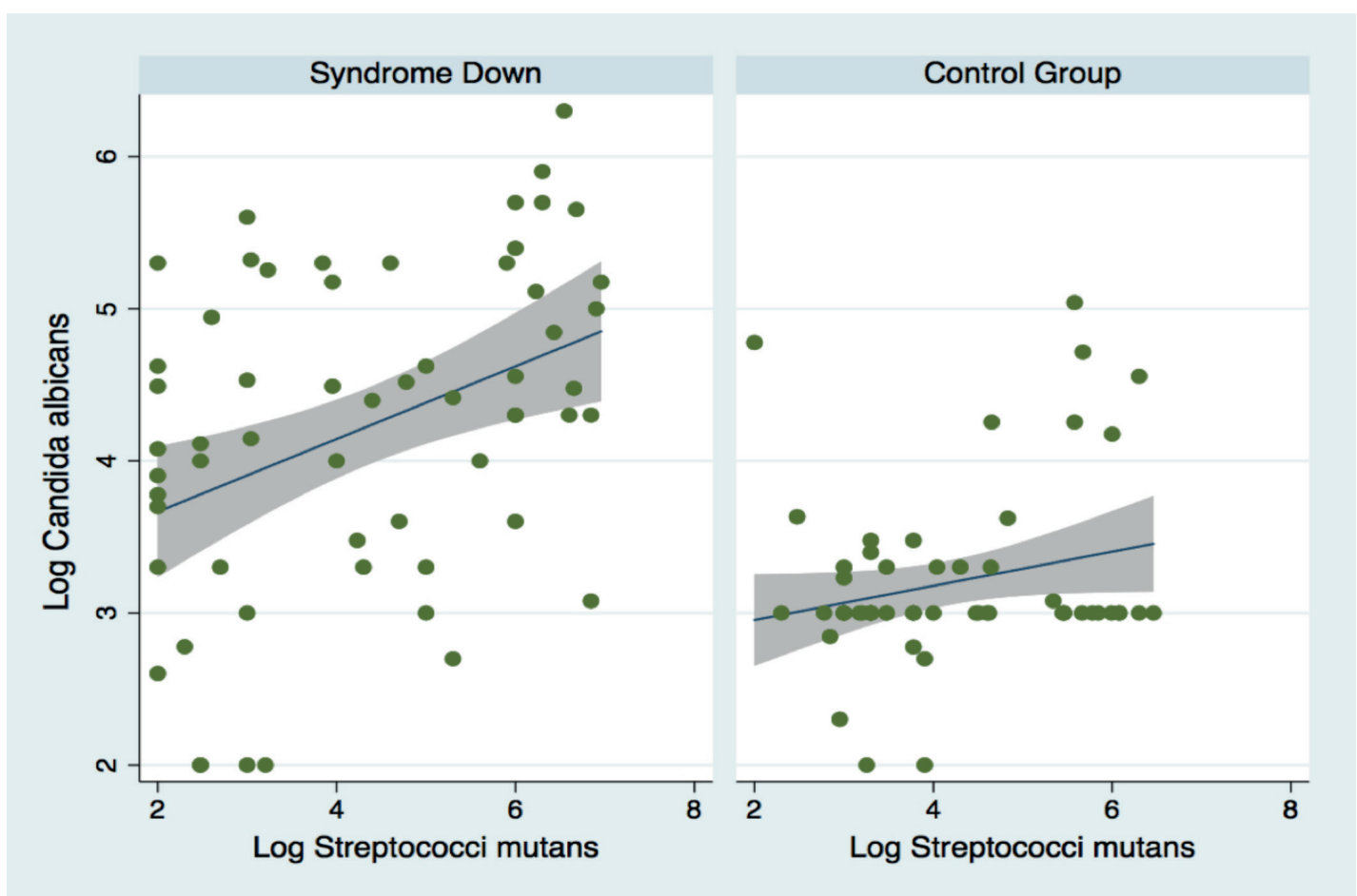

Fig. 1: The patients with Down Syndrome have a highest number of $\log$ C.albicans and of the $\log$ of $\mathrm{ms}$ (colonies/ml) in saliva, this is evidenced with greater slope than in the healthy individuals of the CG. 


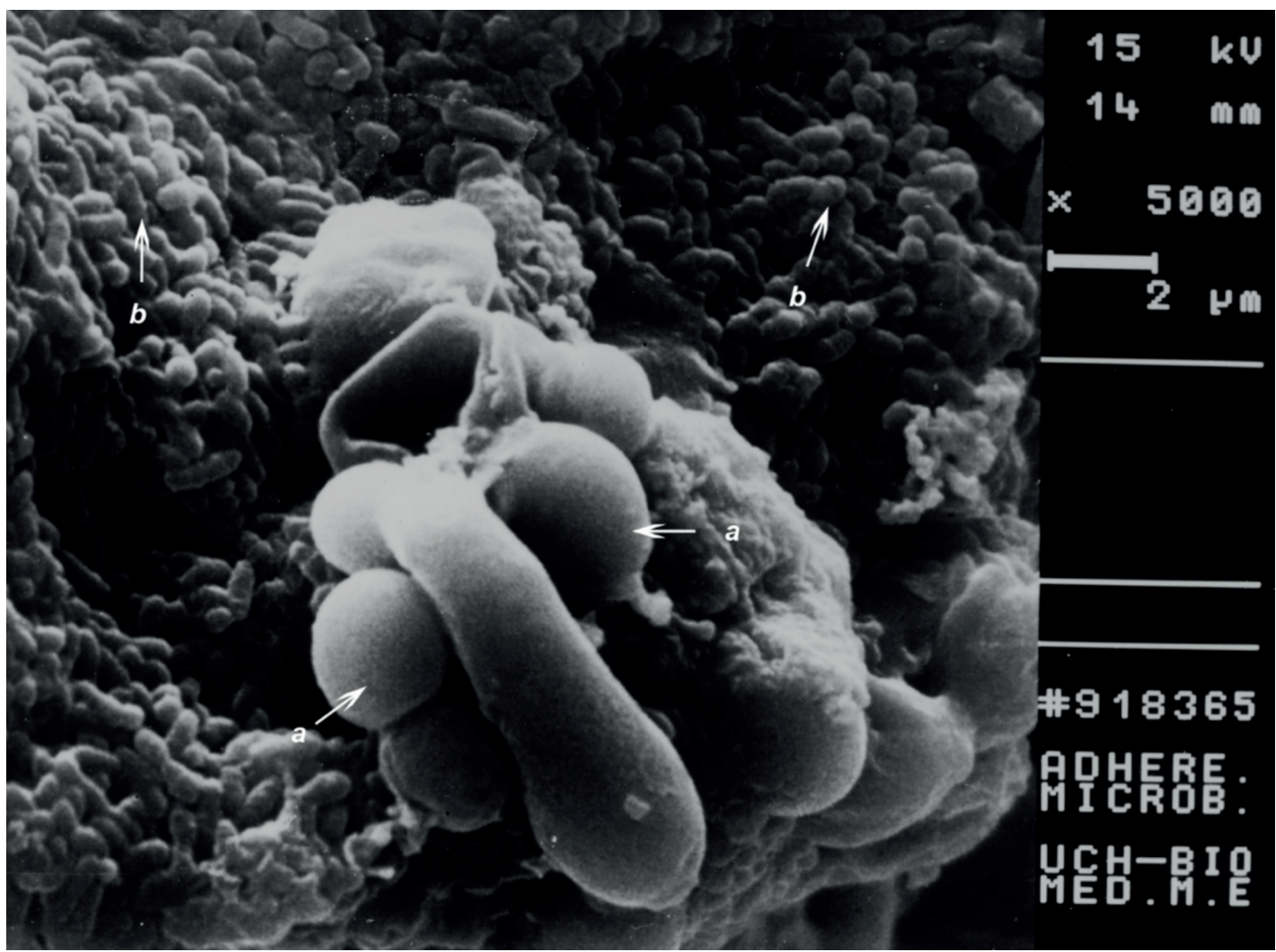

Fig. 2: Scanning electron microscopy of a solid TYCSB culture for ms, where Candida albicans was developed. a) Candida albicans. b) $\mathrm{ms}$.

\section{Discussion}

Patients with Down Syndrome show a greater susceptibility to infections due to several factors that alter immune response as cellular and humoral $(18,19)$. For this reason it is important to take into account the immune response in relation to oral infections, specifically interconnections between microorganisms involved in Caries and Periodontal disease in individuals who present these infectious pathologies, especially in the delivery of new properties that arise from these interrelationships or antagonism between the commensal microorganisms, which cannot be explained from an isolated cell and the possible impact on the patient's systemic level. On the other hand,dental caries in children with mixed dentition is positively correlated with the frequency of oral candidal carriage (25).When interpreting the results obtained according to our hypothesis of the interrelation between $C$ albicans and $\mathrm{ms}$ in saliva of patients in the DS and CG groups, it was evident that in the saliva cultures there were significant differences in the number of $C$. albicans in patients with DS $(P<0.005)(26)$ (Table 1). In other studies conducted in patients with Down syndrome and CG, they showed a high percentage of colonization for C. albicans of $69 \%$, and $35 \%$ for the control group in children, to 7 months, 20 years 6 months (26). In Chile in patients with DS, similar re- sults were obtained for C. albicans (20).

The incidence of C.albicans isolated from the oral cavity, has been reported to be $45 \%$ in neonates, $45 \%$ a $60 \%$ of health children, $30 \%-45 \%$ healthy adults (27), interactions with bacterial present in saliva, as $\mathrm{ms}$ in the DS group. It could be through microbiological studies to study as C.albicans modulates its response from a physical, chemical and metabolic point of view in the presence of other microorganisms (9). Specifically to study the prevalence and possible pathogenicity mechanisms of it, against dental caries in children who are carriers of C.albicans, without leaving aside the set of environmental factors (25).

In both DS and CG groups the ms association was significant for DS, and minor in the control group (Fig. 1). Significant variations were found for $\mathrm{ms}$ in studied age groups (Table 2) but data show no significant diferences for C. albicans in both groups, (Table 3) but C. albicans contributes significantly to the capacity of the dental plaque community to cause disease (8).This would suggest that $C$. albicans could be a nursemaid cell for the ms group. The micro-colonies of S.mutans multiply quickly by doubling their size in the presence of $C$. albicans explaining the possible cause of early caries formation in children. The interaction between C.albicans and S.mutans is complex and have been studied in vi- 
tro model that establish explanations in relation to this duallity.The possible mechanism is that through the production of glucan by $S$. mutans, it binds to the cell wall of $C$. albicans, in the biofilm this supplies sites of adhesion resulting in a greater formation of biofilms of double species (28).

These biofilms of $C$. albicans and $S$. mutans give the possibility of increasing the production of exopolysaccharides (EPS), which allows retain the acid produced by S.mutans. In addition, this structure gives more support to biofilm by increasing the biomass, which only one species could give to house more S.mutans (29). On the other hand, an in vitro study contradicted that the colonization of $C$. albicans is concomitant with $\mathrm{ms}$ as a cariogenic factor, due to the increase in $\mathrm{pH}$ which prevents the loss of minerals (29). Thus C.albicans has often been associated with S.mutans, as they have created an acidogenic microenvironment, in an in vitro study of bacterial culture that incorporate hydroxyapatite discs in the presence of a biofilm of C. albicans and S.mutans, there was evidence of a decrease in the cariogenic potential in terms of acidification and demineralization, so it is proposed that C.albicans would have made a metabolic change like the consumption of oxygen and alkalization within the biofilm by consumption of lactic acid (29). Scientific evidence suggests that DS patients have lower caries prevalence than normal individuals, with risk to bias of reported evidence (14). A study in children with mixed dentition without DS showed that $C$. albicans has a higher prevalence in children with caries than in caries-free children (25). Another shows that C. albicans is associated with S.mutans, in the formation of early caries in children, which in older ages could be colonized only by S.mutans, present in the caries process (13). With the identification of ms serotypes, it has been related that $S$. sobrinus is an aciduric species (Table 2) that is important in the caries of surfaces and rampant caries in children and adolescents with DS, which could be associated with $C$. albicans (12). In relation to serotypes, it was found that in $S$. mutans there is a different serotype corresponding to No- type (21), which could belong to the group $\mathrm{k}$ or other unknown serotype. This no-type serotype was evidenced in DS patients. The serotype No-type and c are in the same amount, while serotype $\mathrm{d}$ is in greater quantity $(p<0.02)$, being that serotype $\mathrm{c}$ the one that has the greatest presence in the human population (12). C. albicans would be significantly associated with the different serotypes of the ms present in humans. It demonstrated the relationship of a mutualism in vivo model between bacteria and fungus that has a clinically severe relevance ubiquitous in infectious diseases. On the other hand, Dentists and General Practitioner should be aware that oral disease can influence the systemic health (30).

Fig. 2 shows an in vitro interrelation between these mi- croorganisms in a TYCSB medium in the groups studied. This medium is selective but not specific for the isolation of $\mathrm{ms}$, and is used to correlate the degree of prevalence of dental caries in humans, being the $S$. mutans and $S$. sobrinus as initiators of enamel caries. The evidence shows that it could increase the number of ms colonies in this medium, and it makes possible the visualization of colonies of $C$. albicans.

In summary the present study shows, in saliva culture, the commensalism between $S$. mutans and $C$. albicans in the oral cavity among CG patients and DS. This commensalism would be an interaction between $C$. albicans and $\mathrm{ms}$ with different serotypes of the group.

\section{References}

1. Aas JA, Paster BJ, Stokes LN, Olsen I, Dewhirst FE. Defining the normal bacterial flora of the oral cavity. J Clin Microbiol. 2005;43:5721-32.

2. Human microbiome project consortium. Structure, function and diversity of the healthy human microbiome. Nature. 2012;486:207-14. 3. Hojo K, Nagaoka S, Ohshima T, Maeda N. Bacterial interactions in dental biofilm development. J Dent Res. 2009;88:982-90.

4. Jakubovics NS, Kolenbrander PE. The road to ruin: the formation of disease-associated oral biofilms. Oral Diseases. 2010;16:729-39.

5. Linossier A, Gajardo M, Olavarria J. Paleomicrobiological study in dental calculus: Streptococcus mutans. Scanning Microscopy. 1996;10:1005-13.

6. Lamfon H, Porter SR, Mccullough M, Pratten J. Formation of Candida albicans biofilms on non-shedding oral surfaces. Eur J Oral Sci. 2003;111:465-71.

7. Zijnge V, Van Leeuwen BM, Degener JE, Abbas F, Thurnheer T, Gmür R, et al. Oral biofilm architecture on natural teeth. PLos One. 2010;5:e9321.

8. Baker JL, Bor B, Agnello M, Shi W, He X. Ecology of the oral microbiome: Beyond bacteria. Trends Microbiol. 2017;25:362-74.

9. Morales DK, Hogan DA. Candida albicans interactions with bacteria in the context of human health and Disease. Plos Pathog. 2010;6:e1000886.

10.Nakano K, Nomura R, Nakagawa I, Hamada S, Ooshima T. Demonstration of Streptococcus mutans with a cell wall polysaccharide specific to a new serotype, $\mathrm{k}$, in the oral cavity. J Clin Microbiol. 2004;42:198-202.

11. Loesche WJ. Role of Streptococcus mutans in human decay. Microbiol Rev. 1986;50:353-80.

12. Devon LA, Hubertine MEW, JAMS J, Vicent MB, Brian MP, Mark ES, et al. Candida -Bacteria: interactions: Their impact on human disease. Am Soc Microbiol. 2016;4:1-19.

13. Duda TD, Angelo GL, Martins CC, Paiva SM, Pordeus IA, Borges-Oliveira AC. Association between dental caries and Down syndrome: A systematic review and meta-analysis. Plos One. 2015;10:1-7. 14. Cahill TJ, Baddour LM, Habid G, Hoen B, Salaun E, Pettersson $\mathrm{GB}$, et al. Challenger in infective endocarditis. J Am Coll Cardiol. 2017;69:325-44.

15. Nakano K, Yoshioka H, Shudo Y, Hata H, Toda K, Taniguchi K, et al. Detection of novel serotype $\mathrm{k}$ Streptococcus mutans in infective endocarditis patiens. J Clin Microbiol. 2006;44:3313-17.

16. Nomura R, Naka S, Nemoto H, Inagaki S, Taniguchi K, Ooshima $\mathrm{T}$, et al. Potential involvement of collagen-binding protein of Streptococcus mutans in infective endocarditis. Oral Dis. 2013;19:387-93. 17. Liu Y, Fillers SG. Candida albicans ALs; a multifunctional adhesin and invasin. Eukaryot Cell. 2011;10:168-73.

18. Izumi Y, Sugiyama S, Shinozuka O, Yamazaki T, Ohyama T, Ishikawa I. Defective neutrophil chemotaxis in Down's syndrome patiens and its relationship to periodontal destruction. J Periodontol. 1989;60:238-42. 
19. Annerén G, Magnusson CG, Lilja G, Nordvall SL. Abnormal serum IgG subclass patten in children with Down's syndrome. Arch Dis Child. 1992;67:628-31.

20. Linossier A, Vargas A, Villegas R, Chimenos E. Quantitative relationship between salivary level of Streptococcus mutans and Candida albicans in children with Down's syndrome. Medicina Oral. 2002;7:284-92.

21. Linossier A, Valenzuela CY, Toledo H. Difference of the oral colonization by Streptococcus of the mutans group in children and adolescents with Down syndrome, mental retardation and normal control. Med Oral Patol Cir Bucal. 2008;13:E536-9.

22. Van Palenstein Helderman WH, Ijsseldijk M, Huis in`t Veld JH. A selective medium for the two major subgroup of bacterium Streptococcus mutans isolated from human dental plaque and saliva. Arch Oral Biol. 1983;28:599-60.

23. Linossier A, Vargas A, Zillmann G, Arriagada M, Rojas R, Villegas R. A semi-quantitative method to assess oral infections with Streptococci mutans in preschool Chilean children. Rev Méd Chile. 2003;131:412-18.

24. Hamada S, Masuda N, Ooshima T, Sobue S, Kotani S. Epidemiological survey of Streptococcus mutans among Japanese children. Identification and serological typing of the isolated strain. JPN Microbiol. 1976;20:33-4.

25. Raja M, Hannan KA. Association of oral candida carriage with dental caries in Children. Caries Res. 2010;44:272-6.

26. Carlstedt K, Krekmanova L,Krom BP, Dahllöf G, Ericsson B, Braathen G, Modeer T. Oral carriage of Candida species in children and adolescents with Down's. Int J Paediatric Dent.1996;6:95-00.

27. Akpan A, Morgan R. Oral Candidiasis. PostGrad Med J. 2002;78:455-9

28.Janus MM, Crielaard W, Volgenant CMC, Van der ven $\mathrm{MH}$, Brandt BW. Candida albicans alter the bacterial microbiome of early in vitro oral biofilm. J Oral Microbiol. 2017;9:1270613.

29. Willems MW, Kos K, Jabra-Risk MA, Krom BP. Candida albicans in oral biofilms could prevent caries. Pathog. Dis. 2016;74:ftw039.

30. Falsetta Ml, Klein M, Colonne PM, Scott-Anne K, Gregoire S, Hua Pai Ch, et al. Symbiotic relations between Streptococcus mutans and Candida albicans synergizes virulences of plaque biofilms In vivo. Infect Immun. 2014;82:1968-81.

\section{Acknowledgements}

We thank Dr. Kazuhiko Nakano for his suggestions to this manuscript, and Dr. Robinson Rojas, also Mr. Robert Hutchinson for your help with the correction of this paper.

\section{Funding}

This study received financial contributions from Fondecyt $\mathrm{N}^{\circ}$ 1960842 .

\section{Conflict of interest}

All the authors of this study declare having no conflicts of interest in any way.

\section{Ethics}

This meta-study was done with data already collected, which were obtained by observing ethical protocols at the time chosen. Parents gave their consent in all cases. The competent children gave their consent in oral form and those who refused to undergo the examination were excluded from the sample. 